\title{
SATELLITE IMAGING DIRECTION ANGLES ESTIMATION METHOD BASED ON RATIONAL POL YNOMIAL COEFFICIENTS
}

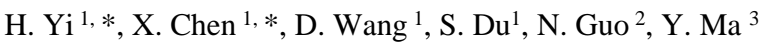 \\ ${ }^{1}$ Academic of Space Information, Space Engineering University, 101416 Beijing, China - (yihui_beijing, laser115, wangdecheng, \\ dushuhan)@163.com \\ ${ }^{2}$ Chinese People's Armed Police Forces Research institute, Beijing, China - sxguonb@163.com

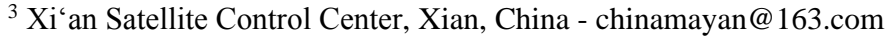

KEY WORDS: Satellite Image, Angle Estimation, Rational Polynomial Coefficient, Azimuth Angle, Incidence Angle, 3D Reconstruction

\begin{abstract}
:
Satellite imaging direction angles, including the azimuth angle and the incidence angle, are the basic information used for satellite camera network structure analysis. They play an important role in 3D reconstruction using satellite images. In this paper, a satellite imaging direction angle estimation method based on rational polynomial coefficients is proposed for use when the satellite imaging direction angles are not available. Using rational polynomial coefficients, a vertical line on the ground is projected into the image plane, and the satellite imaging direction angles are estimated by analyzing the projection. Satellite images acquired by SPOT6, SOPT7 and Pleiades with different satellite imaging direction angles were used to test the feasibility of the proposed method. The experimental results were analyzed in detail combined with the method and the data. The experimental results show that the azimuth angle estimation error is less than 1.30 degrees, and the incidence angle estimation error is less than 0.83 degrees. This level of accuracy is sufficient for satellite camera network structure analysis.
\end{abstract}

\section{INTRODUCTION}

Satellite imaging direction angles, including the azimuth angle and the incidence angle, are used to describe a satellite position relative to a ground object at the moment of imaging. In 3D reconstruction, a stereo pair consisting of two images with different direction angles can be used to reconstruct a 3D model of the ground object (De Franchis et al., 2014; Hu et al., 2016; $\mathrm{Krau} \beta$ et al., 2018). However, the quality of $3 \mathrm{D}$ models obtained from different image pairs varies considerably. There are many factors that affect the quality of $3 \mathrm{D}$ models, but the most important are the view angle between the stereo pair and the incidence angle (Poli et al., 2013; Poli et al., 2015).

According to the research of Facciolo et al. (2017) and Qin et al (2017), to obtain a good 3D model, the pair forming angle should range between 5 degrees and 40 degrees, with the maximum incidence angle below 40 degrees. The best results are obtained with pair forming angles of approximately 20 degrees.

The situation of multiview 3D reconstruction is more complicated (Bosch et al., 2017; Ozcanli et al., 2015). Corresponding to a large number of images with different direction angles, the satellite camera position for each image forms a complex camera network structure. $\mathrm{N}$ images can be combined into $\mathrm{N}(\mathrm{N}-1) / 2$ stereo image pairs. Under the condition that the forming angle of stereo pairs is close to 20 degrees and the maximum incidence angle is below 40 degrees, how to select as few appropriate pairs as possible to cover the face of the ground object is a camera network structure optimization problem (Hepp et al., 2018; Snavely et al., 2006). This is the key to achieving high-quality $3 \mathrm{D}$ reconstruction (Bosch et al., 2017).

The first step in improving the quality of $3 \mathrm{D}$ models is to ascertain the satellite imaging direction angle. Usually, we can obtain satellite imaging direction angles from a physical sensor model (Kim et al., 2007). However, for confidentiality or other reasons, some satellite image venders do not provide a physical sensor model. They provide a rational function model (RFM) as a substitute (Hu et al., 2004; Zhang et al., 2014). Using rational polynomial coefficients (RPCs) attached with an RFM, a large number of 3D reconstruction methods using satellite images have been developed (Beyer et al., 2018; Krauß et al., 2013; Tao, 2002). Due to the advantages of confidentiality and universality, The RFM is gradually becoming a mainstream sensor model for satellite imagery. Almost every satellite image vendor provides a corresponding RPC file while providing a satellite image. However, how to obtain the direction angle from RPCs has not been studied. To improve the performance of $3 \mathrm{D}$ reconstruction methods based on RPCs, we propose a method to estimate satellite imaging direction angles from RPCs in this paper.

\section{METHOD}

As a sensor model, an RFM describes the relative positional relationship between an image and a ground object. This relationship reflects the relative positional relationship between the satellite and the ground object to some extent. Therefore, we can estimate the satellite imaging direction angles using an RFM. Using the RPCs, we project a vertical line on the ground into the image plane, and the satellite imaging direction angles are estimated by analyzing the projection.

\footnotetext{
* Corresponding author
} 


\subsection{Rational Function Model}

The forward form of the RFM defines the ground to image transformation (Tao and $\mathrm{Hu}, 2001)$ :

$$
\begin{aligned}
& r=\frac{p_{1}(X, Y, Z)}{p_{2}(X, Y, Z)} \\
& c=\frac{p_{3}(X, Y, Z)}{p_{4}(X, Y, Z)}
\end{aligned}
$$

where $(r, c)$ and $(X, Y, Z)$ are the normalized image coordinates and ground coordinates, and the range is from -1 to +1 . The normalization of the coordinates is computed using the following equations:

$$
\begin{gathered}
X=\frac{X_{u}-X_{o}}{X_{s}}, Y=\frac{Y_{u}-Y_{o}}{Y_{s}}, Z=\frac{Z_{u}-Z_{o}}{Z_{s}}, \\
r=\frac{r_{u}-r_{o}}{r_{s}}, c=\frac{c_{u}-c_{o}}{c_{s}}
\end{gathered}
$$

where $\left(r_{u}, c_{u}\right)$ and $\left(X_{u}, Y_{u}, Z_{u}\right)$ are the image pixel coordinates and the ground geographic coordinates, $\left(X_{o}, Y_{o}, Z_{o}, r_{o}, c_{o}\right)$ are the offset values for the ground geographic coordinates and the image pixel coordinates, and $\left(X_{s}, Y_{s}, Z_{s}, r_{s}, c_{s}\right)$ are the scale values for the ground geographic coordinates and the image pixel coordinates.

For the polynomials $p_{i}(X, Y, Z)$, the maximum power of each coordinate component $\mathrm{X}, \mathrm{Y}$, and $\mathrm{Z}$ is limited to 3 , and the total powers of all coordinate component of is also limited to 3 . In such a case, each polynomial has 20 cubic terms as follow:

$$
\begin{aligned}
p= & \sum_{i=0}^{m_{1}} \sum_{j=0}^{m_{2}} \sum_{k=0}^{m_{3}} a_{i j k} X^{i} Y^{j} Z^{k}=a_{0}+a_{1} Z+a_{2} Y+a_{3} X \\
& +a_{4} Z Y+a_{5} Z X+a_{6} Y X+a_{7} Z^{2}+a_{8} Y^{2}+a_{9} X^{2} \\
& +a_{10} Z Y X+a_{11} Z^{2} Y+a_{12} Z^{2} X+a_{13} Z Y^{2}+a_{14} Y^{2} X \\
& +a_{15} Z X^{2}+a_{16} Y X^{2}+a_{17} Z^{3}+a_{18} Y^{3}+a_{19} X^{3}
\end{aligned}
$$

where $a_{i j k}$ are the coefficients of the polynomial, called Rational Polynomial Coefficients.

Usually, there are two methods for solving the RPCs (Tao and $\mathrm{Hu}, 2001$; Sheng and Xiao, 2015): 1) terrain-independent method: With the physical sensor models available, the RPCs can be solved using a virtual ground grid with its grid-point coordinates determined using physical sensor model. As it does not need to know the specific terrain, it is called the terrainindependent method. The RPCs solved in this way can be regarded as a kind of fitting to the collinear equation described by the physical sensor model. 2) terrain-dependent method: With no physical sensor model at hand, the 3D virtual ground grid cannot be established. The ground control points (GCPs) collected from maps or digital elevation model (DEM) are used to solve the RPCs. Since GCPs are essentially a description of the terrain, it is called the terrain-dependent method. The RPCs solved by this method can be regarded as fitting to the ground control points. In this case, the solution is highly dependent on the actual terrain, the number of GCPs, and their distribution across the scene. The terrain-dependent solution may cause large error when the GCPs are insufficient or distributed unevenly.
In fact, the RPCs contain the satellite imaging direction information if we consider the RPCs as fitting to the collinear equation. Therefore, the satellite imaging direction angles can be estimated from the RPCs. However, when the RPCs are obtained using the terrain-dependent method, this may introduce a certain error to the estimation of satellite imaging direction angles.

\subsection{Angle Estimation}

Currently, most of the optical mapping satellites use a pushbroom sensor to acquire an image of a ground object. This paper mainly studies the satellite imaging direction angle estimation of images acquired by a push-broom sensor. In fact, the acquisition process of a push-broom image is a multicenter projection. There is no need to accurately estimate the satellite imaging direction angles of each image line, as we just use the angles to analyze the camera network structure. We can use the satellite imaging direction angles of the centerline to represent the whole image when the field of view (FOV) of the satellite is small (Suitable for most high-resolution satellites).

When a satellite is acquiring an image in an inclined pose, its imaging scene can be described as in Figure 1.

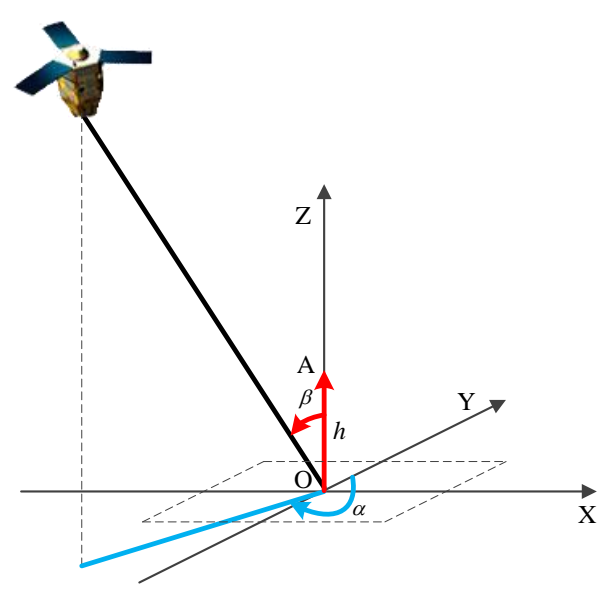

Figure 1. Imaging scene of an inclined satellite

As shown in Figure 1, a right-hand coordinate system is defined by setting the center of the imaging area as the origin; the scanning line direction is set as the $\mathrm{X}$-axis, and the ground normal direction is set as the $\mathrm{Z}$-axis. The incidence angle $\beta$ is defined as the angle between the ground normal and look direction from the satellite. The range for the incidence angle is [0, 90 degrees]. The azimuth angle $\alpha$ is defined as the angle between the Y-axis and the line connect origin and satellite nadir. The range for the azimuth angle is [0, 360 degrees], clockwise positive.

To estimate the satellite imaging direction angles, we set a vertical line OA of height $\mathrm{h}$ (We can take the value $Z_{s}$ ) at the center of the imaging area, as shown in Figure 1. OA can be represented by a series of points with constant $\mathrm{X}, \mathrm{Y}$, and changing $\mathrm{Z}$. Then, we project OA into the image plane using the forward form of the RFM in (1). As a result, we obtain a projection line oa of length $\mathrm{x}$.

The top view of the satellite imaging scene is shown in Figure 2. 


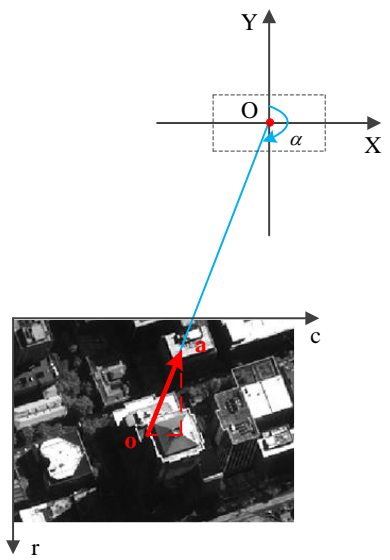

Figure 2. The top view of satellite imaging scene

Although the image plane is not strictly parallel to the ground, it still maintains an approximate parallel relationship. When the accuracy requirement is not high, the angle of the projection line in the image plane can be used to estimate the azimuth angle.

According to Figure 2, we obtain

$$
\tan \left(\alpha-180^{\circ}\right)=-\frac{x_{c}}{x_{r}}=-\frac{n_{c} x_{0}}{n_{r} x_{0}}=-\frac{n_{c}}{n_{r}}
$$

where $x_{c}$ and $x_{r}$ are the projection length of oa in the row direction and column direction respectively, $x_{0}$ is the pixel size, and $n_{c}$ and $n_{r}$ are the pixel length of oa in the row direction and column direction, respectively. Then,

$$
\alpha=180^{\circ}-\arctan \left(\frac{n_{c}}{n_{r}}\right)
$$

With (5), we can estimate the azimuth angle of the satellite by calculating the pixel length of the projection line.

The side view of the satellite imaging scene is shown in Figure 3.

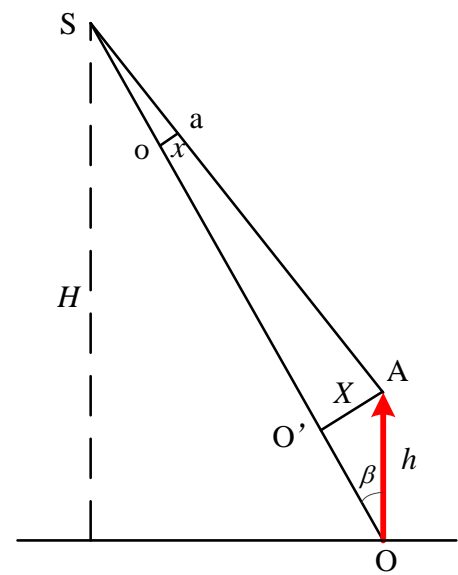

Figure 3. The side view of the satellite imaging scene

Most mapping satellites fly in a sun-synchronous circular orbit. Their orbital altitude is usually several hundred kilometers (approximately 500 700 km) which is much higher than the height of the vertical line. Thus, $\mathrm{SO}^{\prime} \approx \mathrm{SO}$. Make a line passing the point $\mathrm{A}$ and perpendicular to $\mathrm{SO}$ at $\mathrm{O}^{\prime}$.
According to Figure 3, we obtain

$$
\frac{\mathrm{oa}}{\mathrm{So}}=\frac{\mathrm{O}^{\prime} \mathrm{A}}{\mathrm{SO}} \approx \frac{\mathrm{O}^{\prime} \mathrm{A}}{\mathrm{SO}}
$$

Let $\mathrm{AO}^{\prime}=X$. Then,

$$
\frac{x}{f}=\frac{X}{H / \cos \beta}=\frac{h \sin \beta}{H / \cos \beta}=\frac{h}{H} \sin \beta \cos \beta
$$

where $f$ is the camera focal length and $\mathrm{H}$ is the satellite altitude.

For a satellite, there is an imaging formula as follows:

$$
\frac{x_{0}}{f}=\frac{X_{0}}{H}
$$

where $X_{0}$ is the ground sample distance (GSD) at nadir, usually showed as public information. Substituting (8) into (7), we have

$$
\sin (2 \beta)=\frac{2 x H}{h f}=\frac{2 n x_{0} H}{h f}=\frac{2 n X_{0}}{h}
$$

where $n$ is the pixel length of oa, which can be calculated as

$$
n=\sqrt{n_{c}^{2}+n_{r}^{2}}
$$

Then,

$$
\beta=\frac{1}{2} \arcsin \left(\frac{2 n X_{0}}{h}\right)
$$

With (11), we can estimate the incidence angle of the satellite using the pixel length of the projection line.

Table 1 shows the algorithm flow. With the RPCs provided by a vendor, we can estimate the azimuth angle and incidence angle by using the algorithm in Table 1 .

\footnotetext{
Data: RPCs

Result: azimuth angle, incidence angle

1: Set a vertical line of height $h$ at $X_{o}, Y_{o}, Z_{o}$.

2: Project the vertical line to the image plane using forward form of RFM with RPCs.

3: Calculate the length of the projection line in the row direction and column direction, respectively.

4: Estimate the azimuth angle using (5).

5: Calculate the pixel length of the projection line.

6: Estimate the incidence angle using (11).
}

Table 1. Algorithm flow of satellite imaging direction angle estimation

\section{DATASETS}

\begin{tabular}{|c|c|c|c|}
\hline $\begin{array}{c}\text { Data } \\
\text { information }\end{array}$ & Image Type & $\begin{array}{c}\text { Acquisition } \\
\text { date }\end{array}$ & Location \\
\hline SPOT6 & $\begin{array}{c}\text { Stereo } \\
\text { Pushbroom }\end{array}$ & $2016-04-21$ & $\begin{array}{c}\text { Pojravka, } \\
\text { Russia }\end{array}$ \\
\hline SPOT7 & $\begin{array}{c}\text { Tristereo } \\
\text { Pushbroom }\end{array}$ & $2017-07-01$ & $\begin{array}{c}\text { La Dorada, } \\
\text { Colombia }\end{array}$ \\
\hline Pleiades & $\begin{array}{c}\text { Tristereo } \\
\text { Pushbroom }\end{array}$ & $2012-02-25$ & $\begin{array}{c}\text { Melbourne, } \\
\text { Australia }\end{array}$ \\
\hline
\end{tabular}

Table 2. Test image data 
To test the accuracy of the proposed method, three groups of satellite images attached with both a physical sensor model and an RFM were selected for experiments. The satellite imaging direction angles obtained from the physical sensor model are used as the true value. We estimate the satellite imaging direction angles from the RPCs and compare them to the true value. The three groups consist of a stereo pair with nadir GSD
$1.5 \mathrm{~m}$ acquired by SPOT6, a triplet with nadir GSD $1.5 \mathrm{~m}$ acquired by SPOT7, and a triplet with nadir GSD $0.5 \mathrm{~m}$ acquired by Pleiades. Table 2 shows the information of the images used in the experiment.

The three groups of images are shown in Figure 4, Figure 5, and Figure 6.

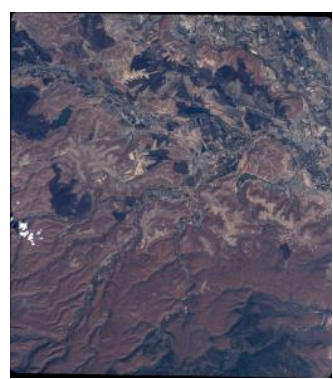

(b) Image B

(a) Image $\mathrm{A}$

Figure 4. Stereo pair of SPOT6

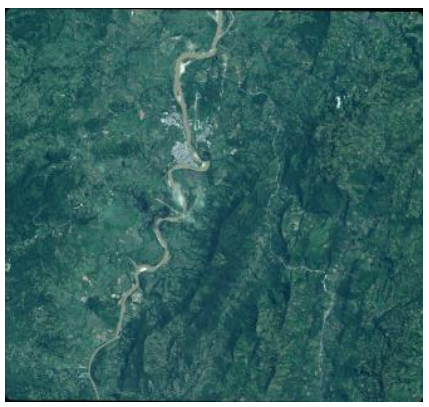

(a) Image $A$

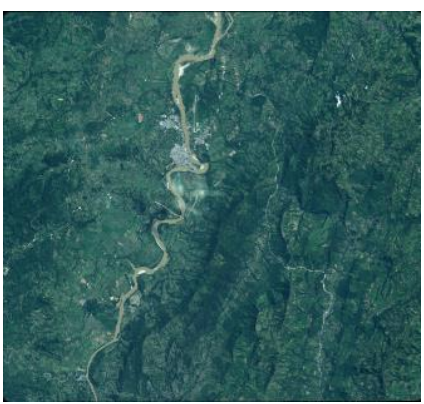

(b) Image B

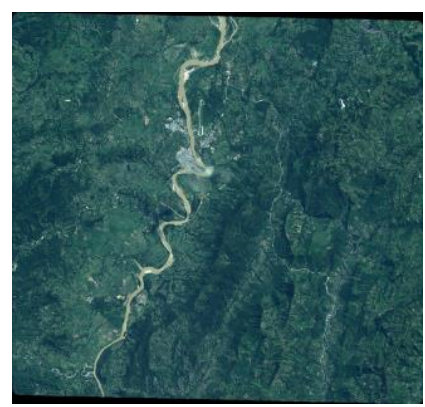

(c) Image $\mathrm{C}$

Figure 5. Tristereo of SPOT7

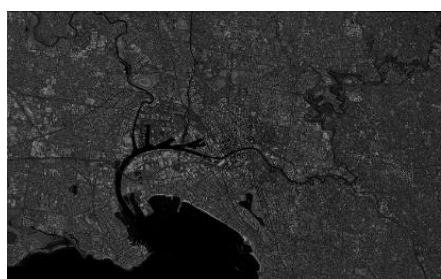

(a) Image $\mathrm{A}$

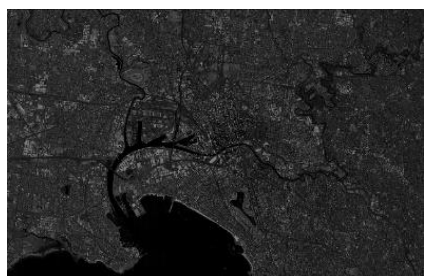

(b) Image B

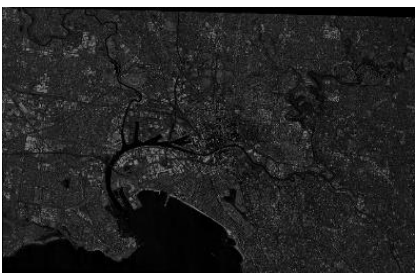

(c) Image $\mathrm{C}$

Figure 6. Tristereo of Pleiades

\section{RESULTS AND DISCUSSION}

The objective of the experiments is to show the feasibility and accuracy of the proposed method. First, the true value of the satellite imaging direction angles are obtained from the physical sensor model. Then, we estimate the satellite imaging direction angles from RPCs using the method proposed in this paper. Finally, we compared the estimation value to the true value and calculated the errors.

The results are shown in Table 3 . The errors are obtained by calculating the absolute difference between the estimation values and the true values. The unit of data is degree.
As shown in Table 3, the maximum of the azimuth angle estimation error is limited to 1.30 degrees, and the mean value of the error is 0.80 degrees; the maximum of the incidence angle estimation error is limited to 0.83 degrees, and the mean value of the error is 0.47 degrees. This accuracy is sufficient for analyzing the camera network structure of multiview satellite images.

In fact, the proposed method is a crude estimation for satellite imaging direction angles, as we did some approximation in the estimation process. This procedure introduced some errors. In the incidence angle estimation, we used GSD as an input. In fact, GSD will change slightly with the change of satellite orbit altitude and the ground elevation. We used GSD as a constant 
for calculation convenience, and this may introduce some error. In the azimuth angle estimation, we used the azimuth angle of the projection line in the image coordinate system as the estimation value of the satellite imaging azimuth angle approximately. This holds for most ortho-ready satellite imagery. For images in acquisition geometry, when the image plane is parallel to the ground plane, this approximation will induce a small error. When the parallel relationship between the image plane and the ground plane is poor, this approximation will induce a large error. In this case, the image should be ortho-rectified first, and the satellite imaging azimuth angle should be estimated by projecting a vertical line into the orthorectified image plane. This will provide better accuracy but requires additional GCPs.

\begin{tabular}{|c|c|c|c|c|c|c|}
\hline \multirow{2}{*}{ Image } & \multicolumn{3}{|c|}{ Azimuth Angle (degree) } & \multicolumn{3}{c|}{ Incidence Angle (degree) } \\
\cline { 2 - 7 } & True & Estimate & Error & True & Estimate & Error \\
\hline SPOT6A & 7.35 & 7.77 & 0.42 & 11.72 & 11.07 & 0.65 \\
\hline SPOT6B & 200.37 & 201.62 & 1.25 & 11.49 & 10.82 & 0.67 \\
\hline SPOT7A & 3.61 & 4.13 & 0.52 & 14.98 & 14.27 & 0.71 \\
\hline SPOT7B & 340.62 & 339.65 & 0.97 & 4.64 & 3.81 & 0.83 \\
\hline SPOT7C & 198.43 & 199.51 & 1.09 & 17.95 & 17.26 & 0.69 \\
\hline PleiadesA & 9.45 & 10.16 & 0.72 & 16.17 & 16.05 & 0.11 \\
\hline PleiadesB & 284.24 & 284.05 & 0.19 & 1.15 & 1.17 & 0.02 \\
\hline PleiadesC & 197.12 & 198.42 & 1.30 & 16.53 & 16.42 & 0.11 \\
\hline Mean & - & - & 0.80 & - & - & 0.47 \\
\hline Max & - & - & 1.30 & - & - & 0.83 \\
\hline
\end{tabular}

Table 3. The results of satellite imaging direction angle estimation

By analyzing the result in Table 3, we find that the accuracy of incidence angle estimation is better than the accuracy of azimuth angle estimation. This is because the error introduced by the approximation of GSD is smaller the error introduced by the parallel approximation.

In addition, the accuracy of the azimuth angle estimation of the three satellites is identical, but the accuracy of the incidence angle estimation is somewhat different. Among them, the estimation error of the incidence angle using the Pleiades triplet images is significantly smaller than the estimation error of the other two groups of images. This is because we used GSD in the incidence angle estimation but not in the azimuth angle estimation. As GSDs of different satellites change in different ranges, the incidence angle estimation of different satellites may show different accuracies.

\section{CONCLUSIONS}

In this study, a practical satellite imaging direction angle estimation method is proposed. The proposed method can recover the satellite imaging direction angle from RPCs. By projecting a vertical line on the ground into the image plane using RPCs, we derived the equations between the length of the projection line and the satellite imaging direction angles and established the relationship between RPCs and the satellite imaging direction angles. Experiments have been performed on various satellite images. The results show that the proposed method was successful in satellite imaging direction angle estimation. The maximum of the azimuth angle estimation error is limited to 1.30 degrees. The maximum of the incidence angle estimation error is limited to 0.83 degrees. This estimation accuracy is sufficient for camera network structure analysis of multiview satellite images.

\section{REFERENCES}

Beyer, R.A., Alexandrov, O. and McMichael, S., 2018. The Ames Stereo Pipeline: NASA's Open Source Software for Deriving and Processing Terrain Data. Earth and Space Science, 5(9): 537-548.
Bosch, M., Kurtz, Z., Hagstrom, S. and Brown, M., 2017. A multiple view stereo benchmark for satellite imagery, Applied Imagery Pattern Recognition Workshop, pp. 1-9.

Bosch, M., Leichtman, A., Chilcott, D., Goldberg, H. and Brown, M., 2017. METRIC EVALUATION PIPELINE FOR 3D MODELING OF URBAN SCENES. XLII-1/W1: 239-246.

De Franchis, C., Meinhardt-Llopis, E., Michel, J., Morel, J. and Facciolo, G., 2014. An automatic and modular stereo pipeline for pushbroom images. ISPRS Annals of the Photogrammetry, Remote Sensing and Spatial Information Sciences, 2(3): 49.

Facciolo, G., De Franchis, C. and Meinhardtllopis, E., 2017. Automatic 3D Reconstruction from Multi-date Satellite Images, IEEE Conference on Computer Vision and Pattern Recognition Workshops, pp. 1542-1551.

Hepp, B., Nießner, M. and Hilliges, O., 2018. Plan3d: Viewpoint and trajectory optimization for aerial multi-view stereo reconstruction. ACM Transactions on Graphics (TOG), 38(1): 4 .

Hu, F., Gao, X.M., Li, G.Y. and Li, M., 2016. DEM EXTRACTION FROM WORLDVIEW-3 STEREO-IMAGES AND ACCURACY EVALUATION. International Archives of the Photogrammetry, Remote Sensing \& Spatial Information Sciences, 41.

Hu, Y., Tao, V. and Croitoru, A., 2004. Understanding the rational function model: methods and applications. International archives of photogrammetry and remote sensing, 20(6).

Kim, T., Kim, H. and Rhee, S., 2007. Investigation of physical sensor models for modelling SPOT 3 orbits. The Photogrammetric Record, 22(119): 257-273.

Krauß, T., D Angelo, P. and Wendt, L., 2018. Cross-track satellite stereo for 3D modelling of urban areas. European Journal of Remote Sensing: 1-10. 
Krauß, T., D Angelo, P., Schneider, M. and Gstaiger, V., 2013. The fully automatic optical processing system CATENA at DLR, ISPRS Hannover workshop, pp. 177-181.

Ozcanli, O.C. et al., 2015. A comparison of stereo and multiview 3-D reconstruction using cross-sensor satellite imagery, IEEE Conference on Computer Vision and Pattern Recognition Workshops, pp. 17-25.

Poli, D., Remondino, F., Angiuli, E. and Agugiaro, G., 2013. Evaluation of Pleiades-1a triplet on Trento testfield. International Archives of Photogrammetry, Remote Sensing and Spatial Information Sciences, XL-1 W, 1: 287-292.

Poli, D., Remondino, F., Angiuli, E. and Agugiaro, G., 2015. Radiometric and geometric evaluation of GeoEye-1, WorldView-2 and Pléiades-1A stereo images for 3D information extraction. ISPRS Journal of Photogrammetry and Remote Sensing, 100: 35-47.

Qin, R., 2017. Automated 3D recovery from very high resolution multi-view satellite images, ASPRS (IGTF) annual Conference, pp. 10.

Snavely, N., Seitz, S.M. and Szeliski, R., 2006. Photo Tourism: Exploring Photo Collections In 3D. Proc of Acm Transactions on Graphics, 25(3): págs. 835-846.

Tao, C.V., 2002. 3D Reconstruction Methods Based on the Rational Function Model. Photogrammetric Engineering \& Remote Sensing, 68(7): 705-714.

Tao, C.V. and Hu, Y., 2001. A Comprehensive Study of the Rational Function Model for Photogrammetric Processing. Photogrammetric Engineering and Remote Sensing, 67(12): $1347-1357$.

Sheng, Q. and Xiao, H., 2015. Satellite Remote Sensing and Photogrammetry. Science Press, Beijing.

Zhang, Y., Liu, J. and Gong, D., 2014. High Resolution Remote Sensing Satellite Application. 2nd Edition. Science Press, Beijing. 